\title{
GAUSSIAN IMAGES OF SURFACES AND ELLIPTICITY OF SURFACE AREA FUNCTIONALS.
}

\author{
D. BURAGO AND S. IVANOV
}

\section{INTRODUCTION}

Before we proceed with rigorous formulations and applications, we want to give a very informal and "easy-to-visualize" description of one of the central results of the paper.

Assume that we are given an $n$-plane $P \subset \mathbf{R}^{N}, n<N$, a collection of oriented $n$-planes $e_{i}, i=1,2, \ldots k$, and a collection of positive reals $a_{i}$, $i=1,2, \ldots k$. We want to know whether there exists an oriented polyhedral surface $S$ such that its boundary belongs to $P$, each of its faces is parallel to one of $e_{i}$ 's (and has the same orientation), and the total area of all faces parallel to $e_{i}$ is $a_{i}$.

If $N=n+1$, the answer is given by the classic Existence Theorem of Minkowski. Let $n_{i}$ be the unit normal to $e_{i}$ (where the orientation of $e_{i}$ determines one of the two choices for a unit normal). Then such a surface $S$ exists if and only if the following obviously necessary "linear algebra" condition is satisfied: $e_{i}$ 's do not lie in a hyperplane and their weighted sum $\sum a_{i} n_{i}$ is normal to $P$. Furthermore, then $S$ can be chosen among convex surfaces.

In general case, already if $n=2$ and $N=4$, no condition like the Minkowski "linear algebra" condition suffices: in generic position $e_{i}$ 's may have no lines in common, so one cannot construct any polyhedral surface of faces parallel to $e_{i}$ 's. Hence we reformulate the problem as follows: given $P$, $e_{i}$ 's and $a_{i}$ 's, we wonder if, for every positive $\varepsilon$, there is a surface such that the total area of its faces parallel to each $e_{i}$ is $a_{i}$, and the total area of all other faces is less than $\varepsilon$.

Now the answer to this question depends on what we exactly mean by a polyhedral surface. If one considers immersed PL-manifolds, we show that, like in the Existence Theorem of Minkowski, such surfaces can always be found provided the obvious "linear algebra" condition similar to that in the theorem of Minkowski is satisfied. However, if one insists on an embedded surface, or at least on an immersed surface whose boundary is embedded in $P$, there are other (not really well understood) constraints.

The first author is partially supported by the NSF Grant DMS-0103739. The second author is partially supported by the CRDF grant RM1-2381-ST-02 and RFBR grants 02-01-00090 and 00-15-96024 . 


\section{Preliminaries and formulations}

The main purpose of the paper is to prove some restrictions or their absence (depending on the set-up) on the (weighted) Gaussian image of a surface of higher codimension. Our motivation to look at these problems came from Finsler geometry, and this work grew from [BI]; however, we believe that these results may be of interest on their own, - and they belong to an area with many open problems. The results can also be applied to studying the ellipticity property of surface area functionals.

2.1. Weighted Gaussian images of surfaces. Let us consider an oriented $k$-dimensional Lipschitz singular chain (possibly with a boundary) in $\mathbf{R}^{n}$. Its weighted Gaussian image is a measure on the oriented Grassmannian $G(k, n)$. It measures "the area of that part of the chain where its tangent plane has certain directions" (a formal definition follows below). Whereas a general definition is given for Lipschitz chains, it is convenient to separate a special case of polyhedral chains.

For a polyhedral chain, its weighted Gaussian image carries the following information: the total area of faces of each (oriented) direction. That is, this is an atomic measure concentrated on the set of directions of faces, with each face of area $s$ and oriented direction $\sigma$ contributing an atom $s \delta(\sigma)$. The corresponding affine-invariant object is a collection of indecomposable $k$ vectors, where all faces parallel to each other and with the same orientation are represented by one $k$-vector parallel to the faces and of the magnitude equal to the total area of the faces.

More formally, for an oriented $k$-dimensional Lipschitz surface $S: \Omega \rightarrow$ $\mathbf{R}^{n}$, its weighted Gaussian image $\mu_{S}$ is the push-forward of the Euclidean surface area on $S$ under the Gaussian map $T: p \mapsto T_{p} S$ from $\Omega$ to the Grassmannian $G(k, n)$ of oriented $k$-planes (the map is defined a. e.) For a (singular) Lipschitz chain with nonnegative coefficients (over $\mathbf{Z}, \mathbf{R}$, or $\mathbf{Q}$ ), the definition is generalized in an obvious way by adding weighted Gaussian images of simplices; that is, if $S=\sum a_{i} S_{i}, S_{i}: \Delta \rightarrow \mathbf{R}^{n}$ (where each $\Delta$ is a standard simplex and all $a_{i}$ 's are nonnegative), we define $\mu_{S}=\sum a_{i} \mu_{S_{i}}$. Now one can always change orientations of some simplices to make all coefficients nonnegative.

Let us remark that all notions and constructions in this paper can be reformulated using an affine-invariant language. Nonetheless, regarding our vector space as $\mathbf{R}^{n}$ and fixing a Euclidean structure turns out to be convenient and makes the exposition and notations less cumbersome. For instance, when considering general area functionals, we may also use the "standard Euclidean surface area" associated with the fixed Euclidean structure.

For a measure $\mu$ on $G(k, n)$, one defines its resulting $k$-vector $\mathbf{E}(\mu)=$ $\int_{G(k, n)} i d \mu$, where $i: G(k, n) \rightarrow \bigwedge^{k}\left(\mathbf{R}^{n}\right)$ is the inclusion of the Grassmannian into the space $\bigwedge^{k}\left(\mathbf{R}^{n}\right)$ of $k$-vectors in the exterior algebra (the inclusion is determined by the Euclidean structure, that is every element of $G(n, k)$ 
is represented by a $k$-vector whose Euclidean $k$-area is 1$)$. If $\mu$ is a weighted Gaussian image of an affine singular $k$-simplex $\sigma$, then $\mathbf{E}(\mu)$ is a decomposable $k$-vector parallel to $\sigma$ and of the same area. We denote this $k$-vector $\mathbf{E}(\mu)$ and also by $\mathbf{E}(\sigma)$. For a $k$-chain $\sigma=\sum a_{i} \sigma_{i}$ where $\sigma_{i}$ are simplices, the resulting vector obviously equals $\sum a_{i} \mathbf{E}\left(\sigma_{i}\right)$.

For a surface $S: \Omega \rightarrow \mathbf{R}^{n}$, the $k$ th exterior power $d^{k} S$ of the differential $d S$ is a $k$-form on $\Omega$ with values in the space of all $k$-vectors $\Lambda^{k}\left(\mathbf{R}^{n}\right)$. The resulting $k$-vector of the weighted Gaussian image of $S$ can be computed as $E\left(\mu_{S}\right)=\int_{\Omega} d^{k} S$. The Stokes' formula implies that the resulting vector of a surface $S$ depends only on its boundary $\partial S$, and we say that $\partial S$ spans the $k$-vector $\operatorname{Span}(\partial S)=\int_{\Omega} d^{k} S$. Note that both $d^{k} S$ and $\operatorname{Span}(\partial S)$ are affine objects.

Similarly, for a chain $S=\sum a_{i} S_{i}, S_{i}: \Delta \rightarrow \mathbf{R}^{n}$, we say that $\partial S$ spans a (possibly non-simple) $k$-vector $\operatorname{Span}(\partial S)=\mathbf{E}\left(\mu_{S}\right)$, or, equivalently, $\operatorname{Span}(\partial S)=\sum \int_{\Delta} d^{k} S_{i}$. It is indeed easy to check that $\operatorname{Span}(\partial S)$ again depends on the boundary $\partial S$ of $S$ only. If the boundary $\partial S$ of $S$ lies in a $k$-plane $P$, then $\operatorname{Span}(\partial S)$ is a simple $k$-vector parallel to $P$. In particular, $\operatorname{Span}(\partial S)=0$ for a closed surface $S$.

There is a subtle difference between chains and surfaces (that is, chains parameterized by manifolds). We will see that all results for chains over $\mathbf{Z}$ can be carried over to the case of surfaces (see Step 2 in the proof of Theorem 2).

\subsection{Measures approximable by weighted Gaussian images of sur-}

faces. The main objective of the paper is to study (the closure of) the class of measures $\mu$ that can arise as weighted Gaussian images of surfaces (or chains) with planar boundaries (that is, when $\partial S$ lies in an affine $k$-plane). We will then show how these results can be applied to a classical question of ellipticity vs. convexity for surface area functionals.

Let us first explain why we want to look at the closure of the class of measures that can be realized as weighted Gaussian images of surfaces, and not at the class itself. First of all, if one has an atomic measure on $G(2,4)$, generically no two 2-planes in the support of the measure have a common line, and hence it is hopeless to construct a polyhedral surface with faces of these directions. We will see that nonetheless one can construct a surface by adding additional faces of arbitrary small total area.

We say that a measure $\mu$ on $G(k, n)$ is realizable by a certain class of surfaces if $\mu$ belongs to the closure of weighted Gaussian images of surfaces from this class. The closure is considered with respect to the weak topology; however, in the polyhedral case our results remain valid for the strong topology as well.

Secondly, consider the following example in already $\mathbf{R}^{3}$. Choose three linearly independent vectors $a, b, d \in \mathbf{R}^{3}$, and a vector $c$ determined by $a+b+c=0$, and consider an atomic measure concentrated on $a \wedge d, b \wedge d$ and $c \wedge d$ with equal weights on each. Geometrically, the faces of a polyhedral 
surface with this Gaussian image are parallel to three planes that have a line in common. It is easy to see that such a surface cannot be closed. However, one can construct a closed surface by adding two arbitrarily small faces not parallel to $d$ (the surface looks like a long prism with two small lids).

Finally, the formulation with the closure is exactly what we need for applications to ellipticity of area functionals.

If we consider a surface whose boundary belongs to $P$, the fact that $\operatorname{Span}(\partial S)$ is parallel to $P$ is a linear constraint on the weighted Gaussian image of $S$. Hence we have a linear constraint on measures realizable by surfaces whose boundaries belong to $P$.

Now we are ready to formulate the main results regarding weighted Gaussian images of surfaces.

Theorem 1 shows that if a measure $\mu$ is such that $\mathbf{E}(\mu)$ is parallel to a $k$ plane $P$, then $\mu$ is realizable by surfaces with boundaries in $P$. Furthermore, we actually show that, given a prescribed boundary $($ a $(k-1)$-cycle $D \subset P$ with $\operatorname{Span}(D)=\mathbf{E}(\mu)), \mu$ is realizable by chains over $\mathbf{R}$ with this boundary.

Theorem 4 demonstrates that there is a measure $\mu$ on $G(2,4)$ with $\mathbf{E}(\mu)$ parallel to $P$ and which cannot, however, be realized by surfaces with nonself-intersecting boundaries in $P$. The Theorem is proven by presenting a specific non-liner constraint on weighted Gaussian images of surfaces with planar non-self-intersecting boundaries.

2.3. Surface area functionals. The proof of Theorem 1 is based on studying a certain artificially introduced surface area functional. In its turn, the Theorems can be applied to studying relationship between ellipticity and convexity of surface area functionals. We will see that the Theorems imply that the question of whether flat regions are area-minimizers with respect to a certain area functional may have different answers for surfaces as opposed to chains over $\mathbf{R}$. Let us introduce necessary definitions and notations.

First we are going to define general (translation-invariant) surface area functionals. They arise from area functions defined on the (decomposable) $k$ vectors in the same way as Riemannian metrics arise from metric tensors by means of integration. In Riemannian geometry, the same term Riemannian metric is used for both the (infinitesimal) metric tensor (allowing to measure the lengths of tangent vectors) and for the functional measuring the lengths of curves. The following definition of a surface area functional associated with an area function on $k$-vectors exactly falls into the same scheme, and we will follow the tradition of Riemannian geometry and use the same term surface area functional for both objects.

Let $G C(k, n)$ denote the Grassmannian cone, that is the set of all decomposable $k$-vectors in $\mathbf{R}^{n}$ ). Let $A$ be a positively homogeneous nonnegative function $A: G C(k, n) \rightarrow \mathbf{R}_{+}$, that is $A(\lambda v)=\lambda A(v)$ for all nonnegative reals $\lambda$ (note that $A(v)$ and $A(-v)$ are not assumed to be equal). We require that the restriction of $A$ to the Grassmannian $G(k, n)$ is bounded (equivalently, $A$ is locally bounded). 
A $k$-dimensional area functional associated with $A$ is a surface area functional $\mathrm{Area}_{A}$ given by the obvious formula

$$
\operatorname{Area}_{A}(S)=\int_{\Omega} A\left(T_{p} S\right) d m(p)
$$

where $S$ is an oriented $k$-dimensional surface $S: \Omega \rightarrow \mathbf{R}^{n}, m$ is the Euclidean surface area, and the tangent space $T_{p} S$ is regarded as a point in $G(n, k)$. Here we again use the inclusion of $G(k, n)$ into $G C(k, n)$ by representing a point of $G(k, n)$ as a $k$-vector of unit Euclidean area. Equivalently, $\operatorname{Area}_{A}(S)=\int_{G(n, k)} A d \mu_{S}$, where $\mu_{S}$ is the weighted Gaussian image of the surface $S$.

An affine-invariant (and rather useless) formula for $\operatorname{Area}_{A}(S)$ reads

$$
\operatorname{Area}_{A}(S)=\int_{\Omega} A \circ d^{k} S\left(\omega^{*}\right) \omega
$$

where $\omega$ is any volume form in $\Omega$. That is, $\omega$ is a positively oriented $k$-form on $\Omega ; \omega^{*}$ is a (dual) $k$-vector field determined by $\omega\left(\omega^{*}\right)=1$; and $A \circ d^{k} S\left(\omega^{*}\right)$ makes sense since the values of $d^{k} S$ are only decomposable $k$-vectors. This formula assumes a very simple form when our surface is an affine $k$-simplex $\sigma: \operatorname{Area}_{A}(\sigma)=A(\mathbf{E}(\sigma))$.

For a Lipschitz chain $S$ with nonnegative coefficients $\left(S=\sum a_{i} S_{i}, S_{i}\right.$ : $\Delta \rightarrow \mathbf{R}^{n}$, where each $\Delta$ is a standard simplex and all $a_{i}$ 's are nonnegative), we define $\operatorname{Area}_{A}(S)=\sum a_{i} \operatorname{Area}_{A}\left(S_{i}\right)$. Now for a general Lipschitz chain, one can always change orientations of some simplices to make all coefficients nonnegative.

2.4. Convexity and ellipticity of surface area functionals. A surface area function $A: G C(k, n) \rightarrow \mathbf{R}$ is said to be convex if it can be extended to a convex function on the vector space $\bigwedge^{k}\left(\mathbf{R}^{n}\right)$ of all $k$-vectors. We define the convex hull $\operatorname{Conv}_{A}$ of $A$ as follows. For a $k$-vector $v$, set $\operatorname{Conv}_{A}(v)=$ $\inf \sum A\left(v_{i}\right)$ for all representations $v=\sum v_{i}$ with $v_{i} \in G C(k, n)$. Then $A$ is convex iff $\operatorname{Conv}_{A}(v)=A(v)$ for all decomposable $k$-vectors $v$. Equivalently, $\operatorname{Conv}_{A}$ is the maximum convex area function not exceeding $A$.

Area $_{A}$ is said to be (semi-)elliptic (with respect to a certain class of chains) if, whenever the boundary $\partial \alpha$ is equal to the boundary of a $k$-disc $D$ embedded into an affine $k$-plane, one has $\operatorname{Area}_{A}(\alpha) \geq \operatorname{Area}_{A}(D)$ (see $[\mathrm{Alm}]$ ). One is particularly interested in ellipticity over $\mathbf{Z}$ and over $\mathbf{R}$ (that is, for the class of chains over $\mathbf{Z}, \mathbf{R}$ ). Sometimes one is interested in ellipticity with respect to chains parameterized by topological discs. This notion is equivalent to ellipticity over $\mathbf{Z}$ if $k>2$ (see [Gr]), whereas in 2 dimensions the situation is rather obscure (see the last paragraph of the paper).

Whereas one often requires strict inequalities in the definition of ellipticity, and the notion we defined should formally be called "semi-ellipticity", we abuse terminology and also call it "ellipticity" for brevity.

Traditionally one also considers ellipticity for surface area functionals that may fail to be translation-invariant. We note that, even though we restrict 
ourselves to translation-invariant area functionals, this does not result in loss of generality. Indeed, both the ellipticity and convexity are infinitesimal notions, and they can be formulated and verified as point-wise conditions on tangent spaces.

For an area function $A: G C(k, n) \rightarrow \mathbf{R}$, define its elliptic hulls $A_{\mathbf{Z}}$ : $G C(k, n) \rightarrow \mathbf{R}$ and $A_{\mathbf{R}}: G C(k, n) \rightarrow \mathbf{R}$ over $\mathbf{Z}$ and $\mathbf{R}$ as follows: for a decomposable $k$-vector $v, A_{\mathbf{Z}}(v)$ (resp. $A_{\mathbf{R}}(v)$ ) equals the infimum of $A$-areas of $k$-chains over $\mathbf{Z}$ (resp. $\mathbf{R}$ ) whose boundaries coincide with a boundary of a $k$-simplex $\sigma$ such that $\mathbf{E}(\sigma)=v$. It is easy to see that $A_{\mathbf{Z}}(v)$ and $A_{\mathbf{R}}(v)$ do not depend on the choice of simplex $\sigma$ representing $v, A_{\mathbf{Z}}$ and $A_{\mathbf{R}}$ are positively homogeneous on $G C(k, n)$, and the associated surface area functionals Area $_{A_{\mathbf{Z}}}$ and Area $_{A_{\mathbf{R}}}$ are elliptic over $\mathbf{Z}$ and $\mathbf{Q}$ respectively. Hence $A_{\mathbf{Z}}\left(A_{\mathbf{R}}\right)$ is the maximal elliptic area function (over $\mathbf{Z}$ and $\mathbf{R}$ respectively) not exceeding $A$.

It is obvious that convexity of $A$ implies that $A r e a_{A}$ is elliptic with respect to any class of chains, and that ellipticity over $\mathbf{R}$ implies ellipticity over $\mathbf{Z}$. Equivalently, $A_{\mathbf{z}} \geq A_{\mathbf{R}} \geq \operatorname{Conv}_{A}$ for any $A$.

Now we are ready to explain the meaning of Theorem 3: it implies that, for any surface area functional, convexity is equivalent to ellipticity over $\mathbf{R}$.

Here is an immediate application of this Theorem to symplectic surface area in a normed space (which is now also often referred to as the Holmes-Thompson surface area). Using Busemann's observation that this surface area may fail to be convex for already 2-dimensional surfaces in a 4-dimensional normed space ([BES]), we conclude that it also may fail to be elliptic over $\mathbf{R}$. This means that there exists a surface whose boundary is $N$ times the unit circle in the $x y$-plane (where $N$ is a positive integer), and whose Holmes-Thompson area (of a certain norm) is strictly less than $N$ times the Holmes-Thompson area of the unit disc (in the $x y$-plane).

As an application of Theorem 4, we construct a surface area functional which is non-convex but still elliptic over $\mathbf{Z}$ (see Section 5). This means that ellipticity over $\mathbf{R}$ is not equivalent to ellipticity over $\mathbf{Z}$.

Now we proceed with precise formulations and proofs.

\section{Constructing a surface with prescribed weighted Gaussian IMAGE}

The main objective of this section is to prove the following

Theorem 1. Let $\mu$ be a measure on $G(k, n)$ such that $\mathbf{E}(\mu)=\int_{G(k, n)}$ id $\mu$ is parallel to a $k$-plane $P$. Then, for every neighborhood $U$ of $\mu$ in the space of measures, there exists a measure $\mu^{\prime}$ which can be represented as the weighted Gaussian image of a surface whose boundary belongs to P. In other words, weighted Gaussian images of surfaces with boundaries in $P$ are dense in all measures satisfying the linear constraint $\mathbf{E}(\mu) \| P$. In particular, weighted Gaussian images of closed surfaces are dense in all measures with $\mathbf{E}(\mu)=0$. 
This Theorem follows from its strengthened polyhedral version, which reads:

Theorem 2. Given a finite collection of unit decomposable $k$-vectors $\alpha_{i}$ and positive reals $a_{i}$ with $\sum a_{i} \alpha_{i}=a \alpha$, where $a \geq 0$ and $\alpha$ is also a unit decomposable $k$-vector, and a positive $\varepsilon$, there exists a polyhedral surface $S$ such that

1. Its boundary $\partial S$ belongs to an affine $k$-plane $P$ parallel to $\alpha$ (if $a=0$, $\partial S=\emptyset$, and we get a closed surface $S$ ),

2. The faces of $S$ can be divided into two subsets. The total area of the faces from the first subset is less than $\varepsilon$; each face from the second subset is parallel to and has the same orientation as one of $\alpha_{i}$; and the total $k$-area of all faces parallel to and with the same orientation as $\alpha_{i}$ is equal to $a_{i}$.

The second condition of the Theorem means that the weighted Gaussian image of $S$ is $\varepsilon$-close (in the norm of the strong topology) to the atomic measure $\sum a_{i} \delta_{\tilde{\alpha}_{i}}$, where $\tilde{\alpha}_{i}$ is the point of the Grassmannian $G(n, k)$ (an oriented $k$-plane) parallel to $\alpha_{i}$.

We will obtain the Theorem as a corollary of the next Theorem 3. The latter asserts that, for any area functional, convexity is equivalent to ellipticity over $\mathbf{R}$.

Recall that, given a positively homogeneous function $A: G C(k, n) \rightarrow \mathbf{R}$, one defines the corresponding area functional (denoted by $A r e a_{A}$ ), and the convex hull $\operatorname{Conv}_{A}$ defined as $\operatorname{Conv}_{A}(v)=\inf \sum A\left(v_{i}\right)$ for all representations $v=\sum v_{i}$ with $v_{i} \in G C(k, n)$. Recall also that, if the boundary of a surface belongs to an affine $k$-plane, then the $k$-vector $\operatorname{Span}(\partial S)=\int d^{k} S$ spanned by the boundary of the surface is a decomposable $k$-vector parallel to the plane. It is obvious that the area of $S$ is no less than $\operatorname{Conv}_{A} \operatorname{Span}(\partial S)$. The following Theorem asserts that (if the boundary is allowed to have self-intersections) this inequality is sharp.

Theorem 3. If an area functional is elliptic over $\mathbf{R}$, then it is convex. Equivalently, for an area functional, its elliptic hull over $\mathbf{R}$ is equal to its convex hull.

Proof. Let $A: G C(k, n) \rightarrow \mathbf{R}$ be an elliptic (over $\mathbf{R}$ ) $k$-dimensional area functional in the space $V=\mathbf{R}^{n}$.

Let $S_{k}(V)$ be the quotient of the vector space of simplicial (PL) $k$-chains over $\mathbf{R}$ in $V$ by the subspace generated by degenerate $k$-simplices and boundaries of degenerate $(k+1)$-simplices. Equivalently, we consider all simplicial chains and identify every chain with any of its triangulations, and every simplex with (-1 times the same simplex with reversed orientation). We will abuse the terminology and use the term "chain" for elements of $S_{k}(V)$.

The boundary functional $\partial: S_{k}(V) \rightarrow S_{k-1}(V)$ is well-defined. An element of $S_{k-1}(V)$ is a boundary if and only if it is closed (i.e. its boundary equals 0$)$. Let $C_{k-1}(V)$ denote the space of all closed chains in $S_{k-1}(V)$. 
Define a semi-norm Fill $_{A}$ on $C_{k-1}(V)$ as follows:

$$
\operatorname{Fill}_{A}(c)=\inf \left\{\operatorname{Area}_{A}(s): s \text { is a } k \text {-chain, } \partial s=c\right\} .
$$

We call Fill $_{A}$ the minimal filling area of $c$. Since $A$ is $\mathbf{R}$-elliptic, we have $\operatorname{Fill}_{A}(\partial \sigma)=\operatorname{Area}_{A}(\sigma)$ for any affine simplex $\sigma$.

Fix a $k$-dimensional subspace $W$ of $V$ and an orientation of $W$. By $C_{k-1}^{W}(V)$ (resp. $S_{k}^{W}(V)$ ) we denote a linear subspace of $C_{k-1}(V)$ (resp. $S_{k}(V)$ ) of chains whose simplices are parallel to $W$.

Now the proof consists of four steps.

Step 1: construct a linear function $L_{1}: C_{k-1}(V) \rightarrow \mathbf{R}$ with the following two properties:

1. $L_{1}(c) \leq \operatorname{Fill}_{A}(c)$ for all $c$, and

2. $L_{1}(c)=$ Fill $_{A}(c)$ if $c$ is the boundary of a positively oriented simplex parallel to $W$.

We define a linear function $l: S_{k}^{W}(V) \rightarrow \mathbf{R}$ (the "algebraic area") to be $l(c)=A(\mathbf{E}(c))$, if $\mathbf{E}(c)$ is positively oriented, and $-A(-\mathbf{E}(c))$ otherwise. Equivalently, for a positively oriented $k$-simplex $s, l(s)=$ Fill $_{A}(\partial s)$, and then $l$ is extended by linearity to all $k$-chains in $S_{k}^{W}(V)$. It is easy to see that the "algebraic filling area" of a simplex (parallel to $W$ ) is proportional to its signed Lebesgue measure.

Consider the following linear function $L_{0}: C_{k-1}^{W}(V) \rightarrow \mathbf{R}$ : for a $(k-1)$ cycle $c \in C_{k-1}(W)$ let $L_{0}(c)=l(s)$, where $s$ is a (unique) $k$-chain $s \in S_{k}^{W}(V)$ such that $\partial s=c$. That is, $L_{0}(s)$ is the "algebraic filing area" of a chain bounded by $s$.

Lemma 3.1. $\left|L_{0}(c)\right| \leq \operatorname{Fill}_{A}(c)$ for all $c \in C_{k-1}^{W}(V)$.

Proof. An easy covering argument shows that $L_{0}(c)=$ Fill $_{A}(c)$ if $c$ is a boundary of a positively oriented cube parallel to $W$. Then the proof goes as follows: first we show that the inequality $L_{0}(c) \leq \operatorname{Fill}_{A}(c)$ holds if $c$ is a sum of boundaries of cubes parallel to $W$ (but lying in different $k$-planes) and obtained from one another by parallel translations. Then we derive the inequality for all chains $c \in C_{k-1}^{W}(V)$.

1. Let $s=\sum_{i=1}^{m} s_{i}$ where $s_{i}$ are translations of the same cube in $W$, and let $W_{i}$ be the $k$-planes containing $s_{i}$. For a large positive integer $N$, consider the chain $s^{N}=\sum s_{i}^{N}$ where $s_{i}^{N}$ is a cube in plane $W_{i}$ obtained from $s_{i}$ by homothety in $N$ times with respect to the center of $s_{i}$. This chain $s^{N}$ can be covered (that is, represented as the sum) by $N^{k}$ translations of $s$. Hence $\operatorname{Fill}_{A}\left(\partial s^{N}\right) \leq N^{k} \operatorname{Fill}_{A}(\partial s)$.

On the other hand, by adding to $s^{N}$ a number of collars of total area $O\left(N^{k-1}\right)$, one can get a chain whose boundary equals $m \partial s_{1}^{N}$. Thus

$$
\begin{aligned}
& m N^{k} L_{0}\left(\partial s_{1}\right)= m N^{k} \operatorname{Fill}_{A}\left(\partial s_{1}\right)=m \operatorname{Fill}_{A}\left(\partial s_{1}^{N}\right)= \\
&=\operatorname{Fill}_{A}\left(\partial s^{N}\right)+O\left(N^{k-1}\right) \leq N^{k} \operatorname{Fill}_{A}(\partial s)+O\left(N^{k-1}\right) .
\end{aligned}
$$


Hence

$$
L_{0}(\partial s)=m L_{0}\left(\partial s_{1}\right) \leq \operatorname{Fill}_{A}(\partial s)+O(1 / N) .
$$

Passing to the limit as $N \rightarrow \infty$, we get the desired inequality for $c=\partial s$.

2. Suppose the assertion is false; then $l(s)=L_{0}(\partial s)>\operatorname{Fill}_{A}(\partial s)$ for some $s \in S_{k}^{W}(V)$. Fix a sufficiently large cube in $W$, consider a collection of translations of this cube covering all simplices of $s$, and let $\sigma$ be the sum of these translations. Represent $s=\sum a_{i} \sigma_{i}$ where $\left\{\sigma_{i}\right\}$ is a triangulation of $\sigma$ into positively oriented simplices, and let $a=\max \left\{\left|a_{i}\right|\right\}$. Then observe that $a \sigma=s+\sum\left(a-a_{i}\right) \sigma_{i}$, therefore

$a \operatorname{Fill}_{A}(\partial \sigma) \leq \operatorname{Fill}_{A}(\partial s)+\sum\left(a-a_{i}\right) \operatorname{Fill}_{A}\left(\partial \sigma_{i}\right)<l(s)+\left(a-a_{i}\right) l\left(\sigma_{i}\right)=a l(\sigma)$, since $a$ and $a-a_{i}$ are nonnegative. Thus $l(\sigma)>$ Fill $_{A}(d \sigma)$, contrary to the statement proved in the first step. Here we use the fact that that $l\left(\sigma_{i}\right)=$ $\operatorname{Fill}_{A}\left(\partial \sigma_{i}\right)$ by definition, since $\sigma_{i}$ are affine simplices.

Observe that Fill $A_{A}$ is a semi-norm on $C_{k-1}(V)$. Then by the Hahn-Banach theorem, there is a linear function $L_{1}: C_{k-1}(V) \rightarrow \mathbf{R}$ extending $L_{0}$ and such that $L_{1} \leq$ Fill $_{A}$.

Step 2: extend $L_{1}$ to a locally bounded linear function $L$ defined on all $(k-1)$-dimensional simplicial chains.

Define a linear function $L: S_{k-1}(V) \rightarrow \mathbf{R}$ extending $L_{1}$ as follows. For a chain $s$, set $L(s)=L_{1}(C y c l e(s))$ where $C y c l e(s)=\partial(\operatorname{Joint}(s, 0))$. In other words, we extend $L_{1}$ from the space of $(k-1)$-cycles to the space of all $(k-1)$ chains by assigning zero value to all simplices with one of the vertices at the origin. For example, if $k=2$, we set $L([a, b])=L_{0}([a, b]+[b 0]+[0 a])$.

Since $L$ is an extension of $L_{1}$, we have

(1) $L(c) \leq$ Fill $_{A}(c)$ for all closed $(k-1)$-chains $c$, and

(2) $L(c)=$ Fill $_{A}(c)$ if $c$ is the boundary of a positively oriented simplex parallel to $W$.

For every $s \in S_{k-1}(V)$, the function $L(s+v)$ is locally Lipschitz in $v \in V$ where $s+v$ denotes the parallel translation of $s$ by $v$. Indeed, $|L(s)-L(s+v)|$ can be estimated from above by the area of a surface spanning the chain Cycle $(s)-C y c l e(s+v)$. It easy to see how to fill this chain with a "collar" (parameterized by Cycle $(s) \times[0,1]$ ) of area no greater than const $\cdot \operatorname{Area}($ Cycle $(s)) \cdot|v|$.

Step 3: smoothen $L$ in such a way that the relations (1) and (2) persist.

Let $\tilde{L}$ be a convolution (w.r.t. the parallel translations) of $L$ with a smooth function $K$ on $V$ (and of total integral 1). That is,

$$
\tilde{L}(c)=\int_{V} L(c+v) K(v) d v
$$


where $c+v$ is the translation of $c$ by vector $v \in V$. The above properties (1) and (2) of $L$ are inherited by $\tilde{L}$.

Fix an oriented linear $(k-1)$-subspace $P$ of $V$. The absolute value of $L$ is bounded by the $(k-1)$-area multiplied by a locally bounded function (e.g. the distance from the origin times $\sup _{G(n, k)} A$ ). Hence the value of $L$ on chains parallel to $P$ is equal to the integral of a density function $f_{P}: V \rightarrow \mathbf{R}$. That is, if a chain $c$ is a weighted sum of positively oriented simplices $\sum a_{i} c_{i}$, then $L(c)=\sum a_{i} \int_{c_{i}} f_{P}$ with respect to the Lebesgue measure. This function $f_{P}$ is measurable since it can be obtained from Lipschitz functions using arithmetics and limits.

For $\tilde{L}$, one defines a collection of functions $\tilde{f}_{P}$ in the same way as we constructed $f_{P}$. Each $\tilde{f}_{P}$ is obtained from $f_{P}$ by a convolution with a smooth function, and hence each $\tilde{f}_{P}$ is smooth (the dependency on the direction of $P$ is still unclear).

Step 4: turn the family of functions $\left\{\tilde{f}_{P}\right\}$ into a differential $(k-1)$-form on $V$.

The collection of functions $f_{P}$ determines a single function $\omega$ on $(k-1)$ tuples of tangent vectors in $V:$ if $v_{i} \in T_{q} V, i=1,2, \ldots, k-1$, and if $P$ is a $(k-1)$-dimensional linear subspace containing the $V_{i}$ 's, then $\omega\left(v_{1}, v_{2}, \ldots v_{k-1}\right)=$ $f_{P}(q) \cdot S$, where $S$ is the oriented Lebesgue area of the parallelepiped spanned by the $v_{i}$ 's

Obviously $\omega$ is skew-symmetric.

Lemma 3.2. $\omega$ is linear in each argument.

Proof. Suppose the contrary: for some $q \in V$ and $v_{1}, \ldots, v_{k-2}, v_{k-1}, v_{k-1}^{\prime} \in$ $T_{q} V$ one has

$\omega\left(v_{1}, \ldots, v_{k-2}, v_{k-1}+v_{k-1}^{\prime}\right)-\omega\left(v_{1}, \ldots, v_{k-2}, v_{k-1}\right)-\omega\left(v_{1}, \ldots, v_{k-2}, v_{k-1}^{\prime}\right)=\delta \neq 0$.

The $v_{i}$ 's and $v_{k-1}^{\prime}$ are linearly independent (otherwise they lie in a $(k-1)$ dimensional subspace $P$, where $\omega=f_{P} \cdot d V o l$, and the linearity is obvious). Thus the $v_{i}$ 's and $v_{k-1}^{\prime}$ form a basis of a $k$-dimensional linear subspace, and the latter can be regarded as the product of the 2-space spanned by $v_{k-1}$ and $v_{k-1}^{\prime}$, and the $(k-2)$-dimensional space spanned by $v_{1}, v_{2}, \ldots v_{k-2}$

Consider a "prism-shaped" product $Q=T \times S$ where $T$ is the 2 -simplex with edges $E_{1}=-v_{k-1}, E_{2}=-v_{k-1}^{\prime}$ and $E_{3}=v_{k-1}+v_{k-1}^{\prime}$, and $S$ is the parallelepiped spanned by $v_{1}, v_{2}, \ldots v_{k-2}$. Consider the family of $(k-1)$ cycles $\varepsilon \partial Q, \varepsilon>0$, where $T_{q} V$ is identified with $V$ in the standard way, so that the origin of $T_{q} V$ is placed at $\left.q\right)$. We are going to show that $\tilde{L}(\varepsilon \partial Q)=$ $\varepsilon^{k-1} \delta+o\left(\varepsilon^{k-1}\right)$. The boundary $\varepsilon \partial Q$ of each $\varepsilon Q=\varepsilon(S \times T)$ consists of two parts: $\varepsilon(T \times \partial S)$ and $\varepsilon(\partial T \times S)$. The faces of the first part $\varepsilon(T \times \partial S)$ split into pairs corresponding to pairs of opposite faces of $S$. Each pair consists of two parallel but oppositely oriented faces at the distance of order $\varepsilon$ from each other. Hence the values of $L$ at these faces cancel out up to 
a term of order $\varepsilon^{k}$, thus $\tilde{L}(\varepsilon(T \times \partial S))=o\left(\varepsilon^{k-1}\right)$. For the second part $\varepsilon(\partial T \times S)=\varepsilon\left(\left(E_{1} \cup E_{2} \cup E_{3}\right) \times S\right)$, we have

$$
\begin{aligned}
& \tilde{L}(\varepsilon(\partial T \times S))=\varepsilon^{k-1} f\left(v_{1}, \ldots, v_{k-2}, v_{k-1}+v_{k-1}^{\prime}\right)- \\
& \quad-\varepsilon^{k-1} f\left(v_{1}, \ldots, v_{k-2}, v_{k-1}\right)-\varepsilon^{k-1} f\left(v_{1}, \ldots, v_{k-2}, v_{k-1}^{\prime}\right)+o\left(\varepsilon^{k-1}\right) \\
& =\delta \varepsilon^{k-1}+o\left(\varepsilon^{k-1}\right)
\end{aligned}
$$

(recall that $E_{1}, E_{2}, E_{3}$ are the sides of the triangle $T$ ).

It follows that $|L(\varepsilon \partial Q)| \geq$ const $\cdot \varepsilon^{k-1}$ for small $\varepsilon$. On the other hand, since $|L| \leq$ Fill $_{A}$ on closed chains, we have $L(\varepsilon \partial Q)=O\left(\varepsilon^{k}\right)$. Contradiction.

Now we are ready to conclude the proof of the Theorem. Since $\omega$ is linear in each argument, and it is smooth on coordinate $(k-1)$-tuples, it is a smooth differential $(k-1)$-form. Consider the $k$-form $d \omega$. Then $\tilde{L}$ of the boundary of a simplex is equal to the integral of $d \omega$ over the simplex. Fix a point $q \in V$ and consider small $k$-simplices near $q$. The inequality $\tilde{L} \leq$ Fill $_{A}$, along with the fact that $\operatorname{Fill}_{A}(\partial \sigma)=\operatorname{Area}_{A}(\sigma)$ for every $k$-simplex $\sigma$, imply that $d \omega_{q} \leq A$ on the Grassmannian cone with equality at decomposable $k$-vectors parallel to $W$. So $d \omega_{q}$, regarded as a linear function on $\bigwedge^{k} V$, is a supporting function for $A$ at the point corresponding to $W$. The convexity of $A$ follows.

To see that the elliptic hull of a surface area functional is equal to its convex hull (the second statement of the Theorem), one argues in the standard way: the elliptic hull is elliptic, and hence convex (by the first statement of the Theorem); on the other hand, the elliptic hull is always greater than or equal to the convex hull; finally, the convex hull is the maximum of convex functions that are bounded from above by $A$ on $G K(n, k)$. Combining these inequalities, one gets the desired equality.

Proof of Theorem 2. The proof consists of two steps:

Step 1: first we construct a chain $\tilde{S}$ satisfying the requirements of the Theorem (for $\varepsilon^{\prime}=\varepsilon / 2$ ) and whose boundary is the boundary is a given simplex $\sigma$ of area $a$ in $W$;

Step 2: we modify $\tilde{S}$ to get a desired surface $S$ (that is, adding additional faces of area not exceeding $\varepsilon / 2$.

Note that in the case $k=2$, the second step is trivial, because an appropriate multiple of a rational chain can always be parameterized by a two-dimensional PL-manifold.

For brevity, we will say that a surface $S$ satisfying the requirements of the Theorem is "a surface with the data $\sum a_{i} \alpha_{i}=a \alpha$, up to faces of the total area $\varepsilon "$.

Step 1. We are given a finite collection of unit decomposable $k$-vectors $\alpha_{1}, \ldots, \alpha_{N}, \alpha$, and nonnegative coefficients $a_{1}, \ldots, a_{N}$, such that $\sum a_{i} \alpha_{i}=$ 
$a \alpha, a \geq 0$. We will argue by induction on the number of nonzero coefficients among $a_{1}, a_{2}, \ldots, a_{N}, a$.

If $a=0$, this means that we want to construct a closed surface. We want to stay away from this situation. Indeed, if $a=0$, we can choose a non-zero $a_{j}$ and consider the data $1 \cdot\left(-\alpha_{j}\right)=\sum_{i \neq j} \frac{a_{i}}{a_{j}} \alpha_{i}$. Hence it suffices to consider the case $a \neq 0$. Thus we assume that all coefficients $a_{i}$ 's and $a$ are positive.

Choose a subset $\left\{\beta_{1}, \beta_{2}, \ldots, \beta_{M}\right\} \subset\left\{\alpha_{1}, \alpha_{2}, \ldots, \alpha_{N}\right\}$ with the following property: $a \alpha$ can be represented as a linear combination of $\beta_{i}$ 's with positive coefficients in a unique way. Without loss of generality, we assume that $\beta_{i}=\alpha_{i}$ for $i=1,2, \ldots, M$, that is, $\beta_{i}$ 's are the first $M k$-vectors the list of $\alpha_{i}$ 's. Let $a \alpha=\sum b_{i} \beta_{i}$.

Since the representation $a \alpha=\sum b_{i} \beta_{i}$ is unique, a linear map $\Sigma: \mathbf{R}^{M} \rightarrow$ $\bigwedge^{k}\left(\mathbf{R}^{n}\right): \Sigma\left(x_{1}, x_{2}, \ldots x_{M}\right)=\sum x_{i} \beta_{i}$ is injective. Hence there is a constant $\lambda$ such that $\left|\Sigma\left(x_{1}, x_{2}, \ldots x_{M}\right)\right| \geq \lambda \sum\left|x_{i}\right|$. Choose such a $\lambda \leq 1$. Since $\Sigma$ is linear, we have

$$
\left|\Sigma\left(x_{1}, x_{2}, \ldots x_{M}\right)-a \alpha\right|=\left|\Sigma\left(x_{1}, x_{2}, \ldots x_{M}\right)-\Sigma\left(b_{1}, b_{2}, \ldots b_{M}\right)\right|,
$$

and hence

$$
\left|\Sigma\left(x_{1}, x_{2}, \ldots x_{M}\right)-a \alpha\right| \geq \lambda \sum\left|x_{i}-b_{i}\right| .
$$

Introduce the following (area) function $A: G C(k, n) \rightarrow \mathbf{R}: A\left(\beta_{i}\right)=0$, and for every $k$-vector $\sigma$ not parallel to any of the $\beta_{i}$ 's, as well as parallel to a $\beta_{i}$ and of the opposite orientation, we set $A(\sigma)=1$.

Obviously, for the convex hull $\operatorname{Conv}_{A}$ of $A$ we have $\operatorname{Conv}_{A}(a \alpha)=0$. By Theorem 3, for the elliptic hull of $A$ over $\mathbf{R}$, we have $A_{\mathbf{R}}(a \alpha)=0$. Hence, given any $k$-simplex $\sigma^{\prime}$ with $E\left(\sigma^{\prime}\right)=a \alpha$ and any $\delta>0$, there exists a (real) $k$-chain $S$ with $\partial S=\partial \sigma^{\prime}$ and $\operatorname{Area}_{A}(S)<\delta$. We will choose a simplex $\sigma^{\prime}$ later. Set $\delta=\lambda \varepsilon / 5$. Then the total area of faces of $S$ that are not parallel to any of the $\beta_{i}$ 's, cannot exceed $\lambda \varepsilon / 5$.

For each $i=1, \ldots, M$, consider the faces of $S$ parallel to $\beta_{i}$ 's and of the same orientation. Let $x_{i} \beta_{i}$ be the resulting vectors of these faces. By (1), we have

$$
\lambda \sum\left|x_{i}-b_{i}\right| \leq\left|\sum x_{i} \beta_{i}-a \alpha\right| \leq \lambda \varepsilon / 5 .
$$

Hence $\sum\left|x_{i}-b_{i}\right| \leq \varepsilon / 5$.

Now consider two cases:

Case 1: $b_{i} \leq a_{i}$ for all $i=1, \ldots, M$. Then we construct the desired chain as the union of the just constructed chain $S$ (where we set $\sigma^{\prime}=\sigma$, that is we require that the boundary of $S$ is the boundary of the same simplex $\sigma$ ), and a closed chain $S^{\prime}$ with the same collection $\left\{\alpha_{i}\right\}$ of oriented directions of faces and with weights $a_{1}-b_{1}, a_{2}-b_{2}, \ldots, a_{M}-b_{M}, a_{M+1}, \ldots, a_{N}$, up to faces of total area at most $\varepsilon / 2$. The latter surfaces exists by the inductive hypothesis.

Case 2: $b_{j}>a_{j}$ for some $j$. Let $\nu=a_{j} / b_{j}, 0<\nu<1$, be the smallest of the ratios $a_{i} / b_{i}, 1 \leq i \leq M$. Let us divide $\sigma$ into two simplices $\sigma_{1}$ and $\sigma_{2}$ of 
areas $\nu a$ and $(1-\nu) a$ respectively. We now require that the boundary $\partial S$ of $S$ is the boundary of $\sigma^{\prime}=\nu^{-1 / k} \sigma_{1}$, where multiplication by $\nu^{-1 / k}$ means homothety in $\nu^{-1 / k}$ times. Then construct the desired chain as the union of the $\nu^{1 / k} S$ (again, a homothety of $S$ in $\nu^{1 / k}$ times), and a surface with boundary $\partial \sigma_{2}$ and with the data

$$
\begin{aligned}
& \left(a_{1}-\nu b_{1}\right) \alpha_{1}+\cdots+\left(a_{j-1}-\nu b_{j-1}\right) \alpha_{j-1}+0 \cdot \alpha_{j}+ \\
& +\left(a_{j+1}-\nu b_{j+1}\right) \alpha_{j+1}+\cdots+\left(a_{M}-\nu b_{M}\right) \alpha_{M}+ \\
& \quad+a_{M+1} \alpha_{M+1}+\cdots+a_{N} \alpha_{N}=(1-\nu) a \alpha
\end{aligned}
$$

up to faces of total area $\varepsilon / 2$. The latter surface exists by the inductive hypothesis.

Step 2. Now we have constructed a simplicial chain with a given weighted Gaussian image (up to faces of total area at most $\varepsilon$ ) and whose boundary is the boundary of a given simplex. We are going to multiply this chain by a large integer, and then we transform this chain into another chain parameterized by a manifold. (Of course, we want to keep the boundary and obtain a surface with almost the same weighted Gaussian image).

First of all, we approximate $S$ (the chain constructed in Step 1) by a rational chain $S^{\prime}$ with the same boundary, and then choose an integer $Q$ such that $Q S^{\prime}$ is an integer chain. Recall that every integer chain can be parameterized by a PL-pseudo-manifold, and furthermore for a given parameterization of the boundary, it can be extended to a parameterization of the whole chain. We will parameterize the boundary $\partial Q S^{\prime}$ by a disjoint union of $Q(k-1)$-dimensional spheres $M_{1}, M_{2}, \ldots, M_{Q}$, and then extend it to a parameterization $f: M \rightarrow \mathbf{R}^{n}$, where $M$ is a PL-pseudo-manifold with $\partial M=\bigcup M_{i}$.

Let $U$ be a small neighborhood of the $(k-3)$-dimensional skeleton of $M$ such that $M \backslash U$ is a manifold (with boundary $\partial M \cup \partial U$ ). We construct a manifold $\tilde{M}$ from the following building blocks:

- the complement $M \backslash U$;

- a tube $\partial U \times[0,1]$, attached to the first block via the natural identification of $\partial U \times\{1\}$ and $\partial U$;

- a copy $M^{\prime} \backslash U^{\prime}$ of $M \backslash U$, attached to the other end of the tube via the identification $\partial U \times\{0\}$ and $\partial U^{\prime} \simeq \partial U$;

- a disjoint union of $Q k$-dimensional disks, attached to the remaining part $\partial M^{\prime}$ of the boundary of the block $M^{\prime} \backslash U^{\prime}$.

Note that $\partial \tilde{M}=\partial M$.

Now define a map $\tilde{f}: \tilde{M} \rightarrow \mathbf{R}^{n}$ as follows. First, $\tilde{f}=f$ on the first block $M \backslash U$. On the tube, define $\tilde{f}(x, t)=t f(x)$; this means that the tube is mapped to the cone over $f(\partial U)$. Finally, the rest of $\tilde{M}$ is sent to the origin.

Now one easily sees that $\tilde{f}$ parameterizes the same chain as $f$ up to an additional chain of arbitrarily small area, and therefore defines almost the 
same weighted Gaussian image. To complete the proof, consider a suitable homothety of $\tilde{f}$, namely $Q^{-1 / k} \tilde{f}$.

\section{Non-linear constraints for weighted Gaussian images of TWO-DIMENSIONAL SURFACES}

Theorem 4. There exist a measure on $G(4,2)$ whose resulting vector is a decomposable 2-vector, but the measure cannot be approximated (in the weak topology) by weighted Gaussian images of surfaces whose boundaries are simple planar curves.

We will prove the theorem by an explicit example of an atomic measure with three atoms. Consider a standard coordinate Euclidean space $\mathbf{R}^{4}$ with the basis $e_{1}, e_{2}, e_{3}, e_{4}$. Then, for instance, an atomic measure with equal weights concentrated on the oriented planes corresponding to the following three 2 -vectors (of equal magnitude):

$$
\begin{aligned}
& w_{1}=\left(e_{1}+e_{3}\right) \wedge\left(e_{2}+e_{4}\right), \\
& w_{2}=\left(e_{1}-e_{3}\right) \wedge\left(e_{2}-e_{4}\right), \\
& w_{3}=2 e_{4} \wedge e_{3},
\end{aligned}
$$

cannot be approximated by weighted Gaussian images of surfaces whose boundaries are simple curves in the $\left(e_{1}, e_{2}\right)$-plane (even though $v_{1}+v_{2}+v_{3}=$ $\left.2 e_{1} \wedge e_{2}\right)$. In the proof we use another collection of 2-vectors, which is more convenient to deal with and which is affine equivalent to this one.

Recall that in the definition of ellipticity of surface area functionals one considers chains that have the same boundary as a (standard) simplex. Under this condition, restricting ourselves to surfaces with simple planar boundaries is equivalent to considering integer chains.

Proof of Theorem 4. Fix $\varepsilon=10^{-10}$. Define three 2-vectors $v_{1}, v_{2}, v_{3}$ in $\mathbf{R}^{4}$ by the following formulae

$$
\begin{aligned}
& v_{1}=\left(e_{1}+\frac{\varepsilon}{2} e_{3}\right) \wedge\left(e_{2}+\frac{\varepsilon}{2} e_{4}\right), \\
& v_{2}=\left(e_{1}-\frac{\varepsilon}{2} e_{3}\right) \wedge\left(e_{2}-\frac{\varepsilon}{2} e_{4}\right), \\
& v_{3}=\frac{1}{2} \varepsilon^{2} e_{4} \wedge e_{3},
\end{aligned}
$$

Observe that the sum of these vectors equals $2 e_{1} \wedge e_{2}$. Introduce an atomic measure $\mu_{0}$ concentrated on the oriented planes corresponding to $v_{1}, v_{2}, v_{3}$ with weights $\left|v_{1}\right|,\left|v_{2}\right|$ and $\left|v_{3}\right|$ respectively. We are going to show that this measure cannot be approximated by weighted Gaussian images of surfaces whose boundaries are simple curves in the $\left(e_{1}, e_{2}\right)$-plane.

We will say that the coordinate $\left(e_{1}, e_{2}\right)$-plane is horizontal, or the $h$-plane, and the $\left(e_{3}, e_{4}\right)$-plane vertical, or the v-plane. We choose orientations in these two planes so that the basis $\left(e_{1}, e_{2}\right)$ of the h-plane is positive and the 
basis $\left(e_{3}, e_{4}\right)$ of the v-plane is negative. Let $P_{v}$ and $P_{h}$ denote the orthogonal projection operators from $\mathbf{R}^{4}$ to the v-plane and h-plane, respectively.

An oriented plane $T$ is said to be $\varepsilon$-vertical (respectively $\varepsilon$-horizontal) if $\left.P_{v}\right|_{T}$ (resp. $\left.P_{h}\right|_{T}$ ) is an orientation-preserving map (with respect to the chosen orientations of the coordinate planes) and its inverse is $(1+\varepsilon)$-Lipschitz. Observe that the planes corresponding to the 2-vectors $v_{1}$ and $v_{2}$ are $\varepsilon$ horizontal, and the plane corresponding to $v_{3}$ is vertical.

Let $U_{v} \subset G(4,2)$ be the interior of the set of $\varepsilon$-vertical planes $\left(U_{v}\right.$ is a small neighborhood of the "vertical" point in $G(2,4))$, and $U_{m} \subset G(2,4)$ be the complement to the union of the set of $\varepsilon$-vertical and the set of $\varepsilon$ horizontal planes. Observe that $\mu_{0}\left(U_{m}\right)=0$ and $\mu_{0}\left(U_{v}\right)>0$. Let $\mu$ be the weighted Gaussian image of a surface $S$ in $\mathbf{R}^{4}$ whose boundary is a simple curve in the h-plane. The following proposition, which immediately implies that $\mu\left(U_{m}\right) \geq \frac{1}{300} \mu\left(U_{v}\right)$, completes the proof.

Proposition 4.1. Let $S$ be a compact piecewise smooth surface in $\mathbf{R}^{4}$ whose boundary $\partial S$ is a simple curve in the h-plane. Consider the following subsets: $S^{V}$ is the set of points $p \in S$ where $T_{p} S$ is $\varepsilon$-vertical; $S^{H}$ is the set of points $p \in S$ where $T_{p} S$ is $\varepsilon$-horizontal; and finally $S^{M}=S \backslash\left(S^{V} \cup S^{H}\right)$ is the complement.

Then $\left|S^{M}\right| \geq 1 / 300\left|S^{V}\right|$.

We precede a formal proof of the Proposition by a very informal illustration of a very special case, with the entire goal of facilitating understanding the idea of the proof; a reader who would not mind struggling through a series of weakly motivated inequalities may bypass this illustration and proceed directly to the proof.

We want to discuss a situation of a surface $S$ whose $\varepsilon$-vertical parts $S^{V}$ is a disc of radius 1 in the $\mathrm{v}$-plane. We also assume that the center $p$ of this disc is at least distance 2 away from the boundary of the surface. Since our goal is to show that the mixed part $S^{M}$ is not too small, we are arguing by contradiction assuming that it is so small that can be neglected in all considerations.

Consider a ball in the intrinsic metric of $S$ of radius 2 and centered at $p$. This ball is contained in a 2-ball in the ambient Euclidean metric, hence so does its orthogonal projection to the h-plane. Since the boundary of $S$ is a simple curve in the h-plane, the projection of $S$ is a region in the h-plane covered with total multiplicity 1 . If the projection to the h-plane reverses orientation at some point, the point belongs to $S^{M}$, and hence all points except a set of a negligibly small area are covered exactly once. Therefore the area of the projection of the 2-ball is approximately $4 \pi$ or less. Note that the $S^{H}$-part is projected by an almost area preserving map, and hence the area of the annulus between the 1-ball and 2-ball is approximately $4 \pi$ or less (we can neglect the $S^{M}$-part, and there is no $S^{V}$-part there).

Now we look at the family of circles of radii from 1 to 2 forming the annulus. By the co-area formula, the average length of such a circle is 
approximately $4 \pi$ or less. On the other hand, if we project the annulus to the v-plane, we are going to get a set of a very small area. Hence the projection of each of the circles surrounds a region of approximately the same area as the first circle in the family (the one that bounds a disc of radius 1 ). By the isoperimetric inequality, we conclude that the length of the projection of every circle is about $2 \pi$ or more. To see a contradiction, it suffices to recall that the average length of a circle is approximately $4 \pi$ or less, whereas the projection shrinks the length in about $1 / \sqrt{\varepsilon}$ times everywhere in $S^{H}$ (and, as usual, we neglect $S^{M}$, and there is no $S^{V}$ in the annulus).

Having this motivating example in mind, we proceed with a formal proof for the general case.

Proof of Proposition 4.1. We may assume that $S$ is an immersed smooth surface (by means of approximation). Then we can freely use the intrinsic metric of the surface (induced by the immersion).

We begin with extending $S$ by adding to it the region in the h-plane outside the boundary of the surface, thus obtaining an unbounded surface coinciding with the $h$-plane everywhere but a compact part. This modification does not change the sets $S^{V}$ and $S^{M}$. In the sequel all notions are considered for the extended surface.

Next we slightly modify the sets $S$ 's and first prove an estimate similar to the one in the Proposition for the new sets. Namely, introduce

$S^{v}=S^{V}$,

$S^{h}$ to be a maximal subset of $S^{H}$ that projects injectively to the h-plane, $S^{m}=S \backslash\left(S^{h} \cup S^{h}\right)$ the complement.

By $B_{r}(p)$ (sometimes $B_{r}$ for brevity) we denote the intrinsic metric ball of radius $r$ centered at $p$. Let $B_{r}^{v}(p)$ (or $B_{r}^{v}$ ), $B_{r}^{h}(p)$ (or $B_{r}^{h}$ ), and $B_{r}^{m}(p)$ (or $B_{r}^{m}$ ) be the intersections of $B_{r}(p)$ with $S^{v}, S^{h}$ and $S^{m}$ respectively.

We need one more constant $a=\frac{1}{100}$.

Lemma 4.2. If $\left|B_{r}^{v}\right| \geq a \pi r^{2}$ and $\left|B_{2 r}^{v}\right| \leq a \pi(2 r)^{2}$, then $\left|B_{2 r}^{m}\right| \geq \frac{a}{2} \pi r^{2}$.

Proof. We need the following estimate: for any $\rho>0$,

$$
\left|B_{\rho}^{h}\right| \leq \pi \rho^{2}(1+\varepsilon)^{2} \leq 2 \pi \rho^{2} .
$$

To prove it, observe that the set $B_{\rho}^{h}$ is contained in the ball of radius $\rho$ in the ambient Euclidean metric, it projects injectively to the h-plane, and the projection multiplies the area by a factor of at least $(1+\varepsilon)^{-2}$.

Introduce the following notation: let $L^{v}(t), L^{h}(t)$ and $L^{m}(t)$ be the lengths of the intersections of the intrinsic metric circle of radius $t$ (centered at $p$ ) with the sets $S^{v}, S^{h}$ and $S^{m}$ respectively. By the co-area formula, $\int_{0}^{2 r} L^{v}(t) d t=\left|B_{r}^{v}\right|$, and similarly for $L^{h}$ and $L^{m}$.

Reasoning by contradiction, assume that $\left|B_{2 r}^{m}\right| \leq \frac{a}{2} \pi r^{2}$. For every $t \in$ $[r, 2 r]$, consider the (oriented) area $A^{v}\left(B_{t}\right)$ of the projection of the intrinsic ball $B_{t}$ to the v-plane. We have

$$
A^{v}\left(B_{t}^{v}\right) \geq A^{v}\left(B_{r}^{t}\right) \geq a \pi r^{2}(1+\varepsilon)^{-2} \geq a \pi r^{2}(1-2 \varepsilon)
$$


from the assumption on $B_{r}^{v}$, and

$$
\left|A^{v}\left(B_{t}^{m}\right)\right| \leq\left|B_{t}^{m}\right| \leq\left|B_{2 r}^{m}\right| \leq \frac{a}{2} \pi r^{2},
$$

and

$$
\left|A^{v}\left(B_{t}^{h}\right)\right| \leq \varepsilon\left|B_{t}^{h}\right| \leq \varepsilon\left|B_{2 r}^{h}\right| \leq 8 \pi \varepsilon r^{2}
$$

by (2). Here we used the fact that the projection $P_{v}$ restricted to an $\varepsilon$ horizontal plane is a $\sqrt{\varepsilon}$-Lipschitz map. Thus

$$
A^{v}\left(B_{t}\right) \geq A^{v}\left(B_{t}^{v}\right)-\left|A^{v}\left(B_{t}^{m}\right)\right|-\left|A^{v}\left(B_{t}^{h}\right)\right| \geq \pi r^{2}(a / 2-10 \varepsilon) .
$$

Then, by the isoperimetric inequality, the length of the projection of the boundary of $B_{t}$ is at least $2 \pi r \sqrt{a / 2-10 \varepsilon}$.

Hence $L^{v}(t)+L^{m}(t)+\sqrt{\varepsilon} L^{h}(t) \geq 2 \pi r \sqrt{a / 2-10 \varepsilon}$. Integrating from $r$ to $2 r$, and using the integration from 0 to $2 r$ in the left-hand side of the inequality, one gets

$$
\left|B_{2 r}^{v}\right|+\left|B_{2 r}^{m}\right|+\sqrt{\varepsilon}\left|B_{2 r}^{h}\right| \geq 2 \pi r^{2} \sqrt{a / 2-10 \varepsilon} .
$$

On the other hand, $\left|B_{2 r}^{h}\right| \leq 8 \pi r^{2}$ by (2), and one of the conditions of the lemma is the inequality $\left|B_{2 r}^{v}\right| \leq 4 a \pi r^{2}$. Recalling the numerical values for $a$ and $\varepsilon$, one has

$$
\left|B_{2 r}^{m}\right| \geq \pi r^{2}(2 \sqrt{a / 2-10 \varepsilon}-8 \sqrt{\varepsilon}-4 a)>\frac{1}{20} \pi r^{2}>\frac{a}{2} \pi r^{2},
$$

a contradiction.

Let $D S^{v}$ denote the set of density points of $S^{v}$, and for every $p \in S^{v}$, let $r(p)$ be the maximum $r$ such that $\left|B_{r}^{v}(p)\right| \geq a \pi r^{2}$.

We need the following well-known folklore lemma:

Lemma 4.3. If a separable metric space is covered by a collection of open balls, there is a countable sub-collection $B_{r_{i}}\left(p_{i}\right)$ such that it still covers the whole space, and $B_{r_{i} / 3}\left(p_{i}\right)$ do not overlap.

By applying this lemma to the covering of $D S^{v}$ by the balls $B_{6 r(p)}(p)$, we get a collection of points $p_{i}$ and radii $r_{i}=r\left(p_{i}\right)$ such that

(1) $\left|B_{r_{i}}^{v}\left(p_{i}\right)\right| \geq a \pi r_{i}^{2}$;

(2) $\left|B_{2 r_{i}}^{v}\left(p_{i}\right)\right| \leq 4 a \pi r_{i}^{2}$

(3) $\left|B_{6 r_{i}}^{v}\left(p_{i}\right)\right| \leq 36 a \pi r_{i}^{2}$;

(4) the balls $B_{2 r_{i}}^{v}\left(p_{i}\right)$ do not overlap;

(5) the balls $B_{6 r_{i}}^{v}\left(p_{i}\right)$ cover $D S^{v}$.

By the first two of these conditions, Lemma 4.2 applies to $p=p_{i}$ and $r=r_{i}$, hence

$$
\left|B_{2 r_{i}}^{m}\left(p_{i}\right)\right| \geq \frac{a}{2} \pi r_{i}^{2} \geq \frac{1}{72}\left|B_{6 r_{i}}^{v}\left(p_{i}\right)\right| .
$$

Since the balls $B_{2 r_{i}}^{v}\left(p_{i}\right)$ do not intersect and the balls $B_{6 r_{i}}^{v}\left(p_{i}\right)$ cover $D S^{v}$, we conclude that

$$
\left|S^{m}\right| \geq \frac{1}{72}\left|S_{17}^{v}\right|=\frac{1}{72}\left|S^{V}\right| .
$$


Now we want to get an estimate on $\left|S^{M}\right|$ (rather than on $\left|S^{m}\right|$ ). Recall that $S^{m} \backslash S^{M}=S^{H} \backslash S^{h}$, and $S^{h}$ is a subset of $S^{H}$ which projects bijectively onto the h-plane. We are going to show that the area $\left|S^{H} \backslash S^{h}\right|$ is small. To do this, we project everything orthogonally to the h-plane and estimate the oriented area (with multiplicity) $A^{h}\left(S^{H} \backslash S^{h}\right)$ of the projection of the set $S^{H} \backslash S^{h}$. Since $S$ coincides with the h-plane outside a compact region, the projection $\left.P_{h}\right|_{S}$ is a map of degree 1, i.e., the h-plane is covered by the projection of $S$ with total multiplicity 1 . Since $S^{h}$ projects bijectively (and preserving the orientation), it follows that the set

$$
S \backslash S^{h}=S^{v} \cup S^{m}=S^{V} \cup S^{M} \cup\left(S^{H} \backslash S^{h}\right)
$$

projects to the h-plane with total multiplicity 0 , hence

$$
0=A^{h}\left(S \backslash S^{h}\right)=A^{h}\left(S^{V}\right)+A^{h}\left(S^{M}\right)+A^{h}\left(S^{H} \backslash S^{h}\right) .
$$

Thus

$$
\left|A^{h}\left(S^{H} \backslash S^{h}\right)\right| \leq\left|A^{h}\left(S^{M}\right)\right|+\left|A^{h}\left(S^{V}\right)\right| \leq\left|S^{M}\right|+\varepsilon\left|S^{V}\right| .
$$

Then

$$
\begin{aligned}
\left|S^{m}\right|-\left|S^{M}\right| \leq\left|S^{H} \backslash S^{h}\right| \leq & (1+\varepsilon)^{2}\left|A^{h}\left(S^{H} \backslash S^{h}\right)\right| \leq \\
& \leq(1+\varepsilon)^{2}\left(\left|S^{M}\right|+\varepsilon\left|S^{V}\right|\right) \leq 2\left|S^{M}\right|+2 \varepsilon\left|S^{V}\right| .
\end{aligned}
$$

Thus $3\left|S^{M}\right| \geq\left|S^{m}\right|-2 \varepsilon\left|S^{V}\right|$. Substituting this into (3) and estimating $\frac{1}{72}-2 \varepsilon>\frac{1}{100}$, we finally get

$$
\left|S^{M}\right| \geq \frac{1}{300}\left|S^{V}\right|
$$

This completes the proof of Proposition 4.1 and Theorem 4.

\section{Applications}

We will give two immediate corollaries of the results from the previous sections.

First of all, we show that there exists a non-convex area functional, which is, nonetheless, elliptic over $\mathbf{Z}$.

Theorem 5. There exists a surface area functional generated by a function $A: G C(2,4) \rightarrow \mathbf{R}$ such that Area $_{A}$ is elliptic over $\mathbf{Z}$, but $A$ cannot be extended to a convex function on the whole $\bigwedge^{2}\left(\mathbf{R}^{4}\right)$.

This means that one can find a collection of 2-vectors "violating the triangle inequality" for $\mathrm{Area}_{A}$, but still flats are area-minimizers.

Proof. We use notations from the proof of Theorem 4. We introduce $A_{0}$ : $G C(2,4) \rightarrow \mathbf{R}$ by setting $A_{0}\left(w_{1}\right)=A_{0}\left(w_{2}\right)=A_{0}\left(w_{3}\right)=0$, and $A_{0}(w)=|w|$ whenever $w$ is not positively proportional to any of $w_{1}, w_{2}, w_{3}$. Let $A$ be the elliptic hull over $\mathbf{Z}$ of $A_{0}$. By Proposition 4.1, $A\left(e_{1} \wedge e_{2}\right)>0$ (we use here that every 2-dimensional integer chain can be parameterized by a surface). Hence $A$ is not convex (and it is elliptic by construction). 
Of course, the area functional constructed in the Theorem is very degenerate (in particular, it assumes zero values on non-zero vectors). One can easily fix this by adding to it the standard surface area multiplied by a small constant. Note also that the elliptic hull of an area functional is automatically continuous.

As the other application, we re-prove a result from [BI], where we considered surface area functionals in normed spaces. In a normed space, there are a number of surface area functionals naturally associated to the norm. Perhaps the most important of them is the one that is now usually referred to as the Holmes-Thompson, or symplectic surface area. It was probably considered first by Busemann (see [Th], [Al], [BI], [SW] for details). For the Holmes-Thompson surface area generated by a certain norm in $\mathbf{R}^{4}$, in [BI] we gave an explicit (and rather cumbersome) example of a surface whose boundary is $N$ times the unit circle in the $x y$-plane (where $N$ is a large number), and whose Holmes-Thompson area is strictly less than $N$ times the Holmes-Thompson area of the unit disc (in the $x y$-plane). It had been noticed already by Busemann in the '50s ([BES]) that the Holmes-Thompson surface area may fail to be convex, and now the existence of such a surface follows immediately from Theorem 3. Let us remark, however, that the construction from [BI] produced a surface which is topologically an immersed disc, whereas this implicit argument gives no control on the topology of the surface: it possibly may have handles. It is relevant to remark here that for chains over $\mathbf{Z}$ bounding a 2-disc, it is quite an intriguing problem whether this difference may play any role: in [BI], we show that flat 2-discs are area minimizers (for the Holmes-Thompson area) among all surfaces (with the same boundary) parameterized by a disc. It would be very amazing if there existed a surface with handles whose area is smaller than that of the flat disc, however this is an open problem. It is also not known whether flat discs are area minimizers in higher dimensions. It seems that both directions of generalizing the 2-dimensional result from [BI] encounter very similar difficulties.

\section{REFERENCES}

[Alm] J. Almgren. "Existence and regularity almost everywhere of solutions to elliptic variational problem among surfaces of varying topological type and singularity structure". Math. Ann.Ann. Math. Ser. 2, vol. 87 (1968), pp. 321-391

[Al] J.-C. Alvarez Paiva "Some problems in Finsler Geometry", preprint, 23 pages

[BI] D. Burago, S. Ivanov "Area-minimizing property of flats in normed spaces and asymptotic geometry of tori" To appear in the "Annals of Mathematics"

[BES] H. Busemann, G. Ewald, and S. Shephard "Convex bodies and convexity on Grassmann cones. I-IV", Math. Ann. vol 151, 1963 pp. 1-41

[Gr] M. Gromov "Filling Riemannian manifolds", J. Diff. Geom. vol 18, 1983 pp. 1-147

[SW] R. Schneider, J. A. Wieacker "Integral Geometry in Minkowski Spaces", Advances in Mathematics, vol 129, 1997, pp. 222-260

[Th] A. C. Thompson "Minkowski Geometry", Encyclopedia of Math and Its Applications, Vol. 63, Cambridge Univ. Press. 1996 
Department of Mathematics, The Pennsylvania State University, UniverSITY PARK, PA 16802

E-mail address: burago@math.psu.edu

Steklov Math. Institute, St. Petersburg, Russia

E-mail address: svivanov@pdmi.ras.ru 